\title{
Bronchial Arteries: An Arteriosclerosis-Resistant Circulation
}

\author{
Christophoros Kotoulas ${ }^{\mathrm{a}, \mathrm{b}}$ Maria Melachrinou ${ }^{\mathrm{c}}$ George N. Konstantinou ${ }^{\mathrm{a}}$ \\ Dimitrios Alexopoulos $^{d}$ Dimitrios Dougenis ${ }^{b}$ \\ ${ }^{a}$ Department of Cardiothoracic Surgery, 401 General Military Hospital of Athens, Athens, and Departments of

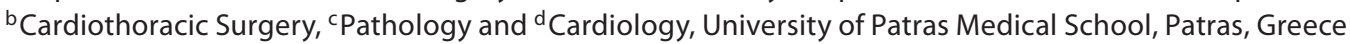

\section{Key Words}

Bronchial arteries $\cdot$ Arteriosclerosis $\cdot$ Coronary arteries •

Mediastinum

\begin{abstract}
Background: Until now, it is unknown whether and to what extent arteriosclerotic disease affects the bronchial arteries. Objectives: We conducted this pilot study to estimate the prevalence of arteriosclerosis of the bronchial arteries, to correlate it with certain clinicolaboratory arteriosclerotic parameters or any coexistent coronary artery disease (CAD) and to validate the clinical significance. Methods: Bronchial arteries $10-15 \mathrm{~mm}$ long were obtained from 40 patients with a mean age of 62.3 years who underwent major thoracic procedures. Their medical history and detailed clinical and laboratory arteriosclerotic risk factors were documented. $\boldsymbol{R e}$ sults: The mean diameter of bronchial artery specimens was $0.97 \mathrm{~mm}$. Histology revealed medial calcific sclerosis only in 1 patient (2.5\%) without simultaneous, established atherosclerotic lesions or narrowing of the lumen. Furthermore, the vessel diameter was significantly correlated not only with the advanced stage of the disease $(p=0.031)$, but also with the proximal occlusion of the bronchial tree $(p=0.042)$. We noted a marginally not significant correlation between arte-
\end{abstract}

riosclerosis and metabolic syndrome $(p=0.075)$, independent from a history of CAD ( $p=0.84)$. Conclusions: Bronchial arteries exhibit only medial calcific sclerosis. CAD and chronic obstructive pulmonary disease do not seem to affect them in terms of atherosclerotic alteration findings or vessel diameter changes. The bronchial resistance to arteriosclerosis might support the mediastinal status quo through their anastomoses, contributing to all its structures, and might be indirect evidence of a different physiological function of the bronchial endothelium, which needs to be further investigated.

Copyright $\odot 2009$ S. Karger AG, Basel

\section{Introduction}

During the past decades, knowledge regarding the function of bronchial arteries has increased considerably [1-3]. However, the presence and the degree of arteriosclerosis affecting the bronchial arteries have yet to be investigated.

Our previous study demonstrated the existence of bronchial-coronary anastomotic routes in the pig [4]. Similar findings have been reported in human autopsy [5], and seldom, in case reports [6]. Furthermore, Riquet

\section{KARGER}

Fax +4161306 1234 E-Mail karger@karger.ch www.karger.com
(ㄷ) 2009 S. Karger AG, Basel 0025-7931/10/0794-0333\$26.00/0

Accessible online at: www.karger.com/res
Christophoros Kotoulas, MD, $\mathrm{PhD}$

38 Kifissias Ave, Ampelokipoi, Athens

GR-11526 (Greece)

Tel. +30 210778 2220, Fax +30 210777 2329, E-Mail info@ kotoulas.com 
et al. $[7,8]$ have demonstrated that the presence of the bronchial-coronary anastomotic route is associated with the presence of severe coronary artery disease (CAD).

The aim of our study is to assess the degree of arteriosclerosis in bronchial artery specimens taken from living donors during major thoracic operations, to investigate whether arteriosclerosis is associated with the presence of CAD, any arteriosclerotic risk factors or coexisting pulmonary disease and to identify its clinical significance.

\section{Patients and Methods}

\section{Material}

In this prospective study, biopsies from bronchial arteries from patients who underwent a major thoracic operation for lung or esophageal cancer at our institution were obtained. Thirtyeight men and 2 women, with a mean age of $62.3 \pm 12.5$ years (range 19-79), took part in the study. The University of Patras Medical School Review Board approved the study, and written consent was obtained from all patients during their preoperative assessment. Thirty-nine patients underwent major lung resection and 1 patient underwent esophagectomy.

Blood testing was performed in all patients for serum glucose, total cholesterol, triglycerides, low-density lipoprotein, high-density lipoprotein, atherosclerotic index, apolipoproteins A1 (apo A1) and B (apo B) and lipoprotein A.

During the preoperative assessment, all risk factors related to arteriosclerosis were recorded for each patient. These included: gender, age, body surface area (calculated using the Mosteller formula), smoking status, alcohol consumption, arterial hypertension, body mass index, diabetes mellitus, hyperlipidemia, CAD, peripheral vascular disease and a positive family history. We also recorded the existence of chronic obstructive pulmonary disease (COPD), the bronchoscopic findings and the presence of calcifications in the thoracic aorta, as they appeared on the CT scan.

Twenty patients underwent pneumonectomy, 6 bilobectomy, 13 lobectomy and 1 patient esophagectomy. Left thoracotomy was performed in 27 patients, and right thoracotomy in the remaining. Mediastinal lymph node dissection was performed in all patients.

\section{Method}

Specimens of the bronchial artery of varying sizes (10-15 mm long) were dissected and excised. For the left lung, this was succeeded by ensuring access very close to the descending aorta just adjacent to the main bronchus. On the right side, this was accomplished after mobilization of the azygos vein, adjacent to the main bronchus. Specimens were stained using hematoxylin and eosin (H\&E). The following parameters were evaluated: the diameter of the vessels, the thickness of the vessel wall, the presence of fibrous tissue of the media or adventitia, the degree of calcification on the media or inner elastic membrane, and the presence of arteriosclerosis.

Based on the above parameters, all the arterial changes were classified as arteriosclerotic (i.e. atherosclerotic or medial calcific sclerotic) or not, including the age-related changes, changes related to hypertension and the nonsignificant changes.
Table 1. Demographic characteristics and medical history

\begin{tabular}{lc}
\hline Age, years & $62.3 \pm 12.5$ \\
Gender, male & $38(95 \%)$ \\
Smoking habits & 29 \\
$\quad$ Active & 5 \\
$\quad$ Ex-smoker & 6 \\
$\quad$ Non-smoker & \\
Body surface area, $\mathrm{m}^{2}$ & $1.82 \pm 0.12$ \\
$\quad$ Mean \pm SD & $1.48-2.02$ \\
$\quad$ Range & 17 \\
Hypertension & 16 \\
Excessive alcohol consumption & 11 \\
COPD & 7 \\
CAD & 6 \\
Diabetes mellitus & 3 \\
Peripheral vascular disease & 10 \\
Family history of arteriosclerosis & \\
\hline
\end{tabular}

Statistical Analysis

All descriptive statistics are presented as the mean \pm standard deviation. The distributions of all variables of interest were initially examined. In non-normally distributed variables, log transformation was found to be the most appropriate to succeed normal distribution. Comparisons between normally distributed parameters were performed using Student's t test; otherwise, for highly skewed variables, the Mann-Whitney test was applied. ANOVA was used for multiple comparisons. All reported values are based on 2-sided tests and compared with a significance level of 5\%. SPSS for Windows, release 12.0.0 (SPSS Inc., Chicago, Ill., USA), was used for all calculations.

\section{Results}

Demographic characteristics and the medical history of the included patients are presented in table 1.

Preoperative bronchoscopy showed no intrabronchial lesion in 13 patients. However, the tumor had obstructed the segmental bronchus in 12 patients, the lobar bronchus in 8 patients, the intermediate bronchus in 3 patients, and there was obstruction of the main bronchus in 4 patients. Furthermore, 11 patients were diagnosed with COPD based on their medical history and spirometry. Last but not least, a chest CT scan demonstrated the presence of calcifications on the thoracic aorta, mainly in the aortic arch, and in the descending aorta in 22 patients.

Seventeen patients $(42.5 \%)$ had raised total cholesterol levels and 8 patients (20\%) had elevated triglyceride levels. Low-density lipoprotein was above the normal range in 20 patients (20\%), and high-density lipoprotein was 
Table 2. Analytical presentation of laboratory results with the corresponding normal values

\begin{tabular}{|c|c|c|c|c|c|}
\hline & Minimum & Maximum & Mean & SD & $\begin{array}{l}\text { Normal } \\
\text { range }\end{array}$ \\
\hline Glucose, mg/dl & 72 & 180 & 104.15 & 25.74 & $70-110$ \\
\hline Cholesterol, mg/dl & 115.0 & 303.0 & 195.42 & 37.84 & $<200$ \\
\hline Triglycerides, mg/dl & 51.0 & 238.0 & 119.20 & 47.95 & $40-160$ \\
\hline $\mathrm{LDL}, \mathrm{mg} / \mathrm{dl}$ & 51.0 & 191.0 & 125.27 & 32.37 & $<160$ \\
\hline HDL, mg/dl & 27.0 & 79.0 & 44.25 & 10.11 & $35-80$ \\
\hline Atherosclerotic index & 2.96 & 7.83 & 4.53 & 1.011 & $<3.5$ \\
\hline apo $\mathrm{A} 1, \mathrm{mg} / \mathrm{dl}$ & 82.0 & 220.0 & 131.48 & 30.97 & $73-169$ \\
\hline apo B, mg/dl & 59.0 & 144.0 & 98.79 & 20.91 & $58-138$ \\
\hline Lipoprotein A, mg/dl & 6.0 & 138.0 & 31.07 & 25.47 & $0-30$ \\
\hline VLDL, mg/dl & 11.0 & 43.0 & 23.14 & 9.49 & $4-32$ \\
\hline Lipids, mg/dl & 406.0 & 887.0 & 583.78 & 116.48 & $450-800$ \\
\hline
\end{tabular}

$\mathrm{LDL}=\mathrm{Low}$-density lipoprotein; HDL = high-density lipoprotein; VLDL = very low-density lipoprotein.

above the normal range in 5 patients (12.5\%). An abnormal atherosclerotic index was found in 34 patients (85\%). apo A1 was above the normal range in $3(10.3 \%)$, while apo $B$ was above the normal range in only 1 patient (3.4\%). Lipoprotein A was above the normal range in 8 patients (27.6\%). Finally, the ratio of apo B to apo A1 was $>1$ in 4 patients. Laboratory results are analytically presented in table 2 with the corresponding normal values.

The tumors were histologically stratified following the World Health Organization criteria and staged according to the Mountain criteria $[9,10]$. All resections were in disease-free margins. There was 1 esophageal adenocarcinoma, 1 lung abscess and 38 non-small cell carcinomas, including squamous cell carcinoma $(\mathrm{n}=23)$, adenocarcinoma $(\mathrm{n}=10)$, typical carcinoid $(\mathrm{n}=3)$, melanoma $(\mathrm{n}=$ $1)$, and neuroendocrine tumor $(\mathrm{n}=1)$. The TNM stage was IA in 3 cases, IB in 5, IIA in 3, IIB in 14, IIIA in 11, IIIB in 2 and IV in only 1 case. Tumor differentiation was poor in 27, moderate in 8 and well in 4 cases.

The mean diameter of the bronchial arteries was 0.93 $\pm 0.34 \mathrm{~mm}$ (range $0.55-1.70$ ), whilst the average thickness of their wall was $0.25 \pm 0.94 \mathrm{~mm}$ (range 0.08-0.42). Significant correlation was found between the thickness and the diameter of the bronchial arteries (Spearman's $\rho=0.642 ; \mathrm{p}<0.001$ ).

Although a CT scan demonstrated that calcifications were present on the thoracic aorta (mainly in the aortic arch and the descending aorta) in 22 patients, only mild deposition of calcium along the inner elastic membrane was observed in 16 cases, out of which 5 were localized deposits and 11 were granular. Furthermore, in the ma- jority of cases (38/40), no calcium deposition was shown in the media, while in 2 cases deposition was localized.

Afterwards, the findings were classified as age-related changes in 32 cases (fig. 1), hypertensive-related changes in 3 (fig. 2), within physiological limits in 4 (fig. 3) and calcific medial sclerosis (Mönckeberg's disease) in 1 case (fig. 4). None of the patients had typical atherosclerosis.

All comparisons between different levels of hypertrophy, fibrosis and calcium deposition in terms of vessel size and thickness of the wall are presented in tables 3 and 4. Mild hypertrophy of the media was shown in 11 cases, moderate in 18 , severe in 3 , moderate concentric in 1 and severe concentric in 2 cases, while in 5 cases no hypertrophy was noted. Overall, there was no difference between the mean diameter and the degree of hypertrophy of the media $(p=0.072)$; however, specimens with severe concentric hypertrophy of the media were found to be significantly larger compared with specimens without any degree of hypertrophy $(\mathrm{p}=0.01)$, but also when compared with those with mild hypertrophy $(\mathrm{p}=0.038)$. However, the thickness of the vessel wall was significantly different among the different degrees of hypertrophy of the media $(p<0.001)$.

In addition, significant differences were found between the thickness of the vessel wall and the degree of fibrosis of the intima $(\mathrm{p}<0.001)$ and of the adventitia $(\mathrm{p}<0.001)$. These strong statistical differences were consistent with all comparisons between the cases without fibrosis and all types of fibrosis ( $\mathrm{p}<0.001)$, as well as with the comparison between cases with moderate and mild fibrosis $(\mathrm{p}<0.01)$ 


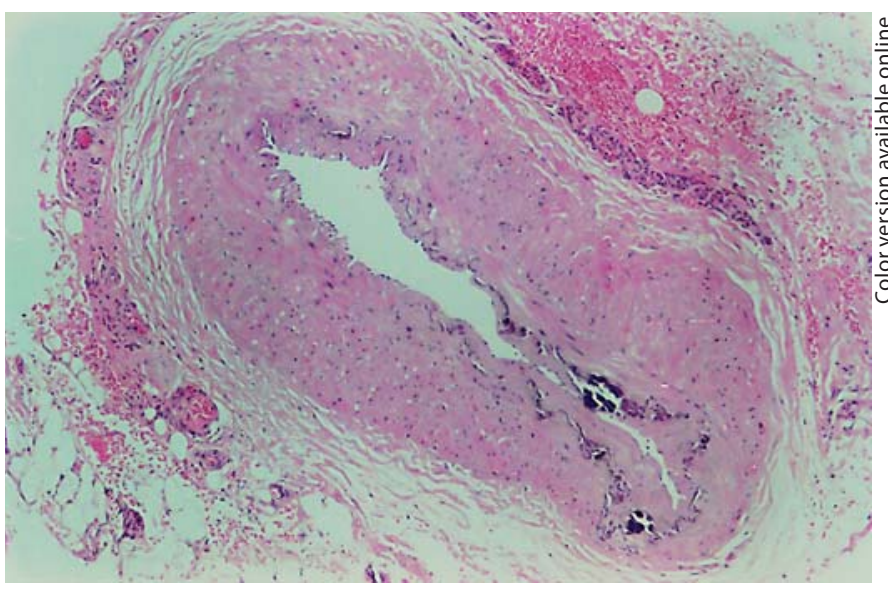

Fig. 1. Bronchial artery specimen with age-related changes. H\&E. $\times 100$.

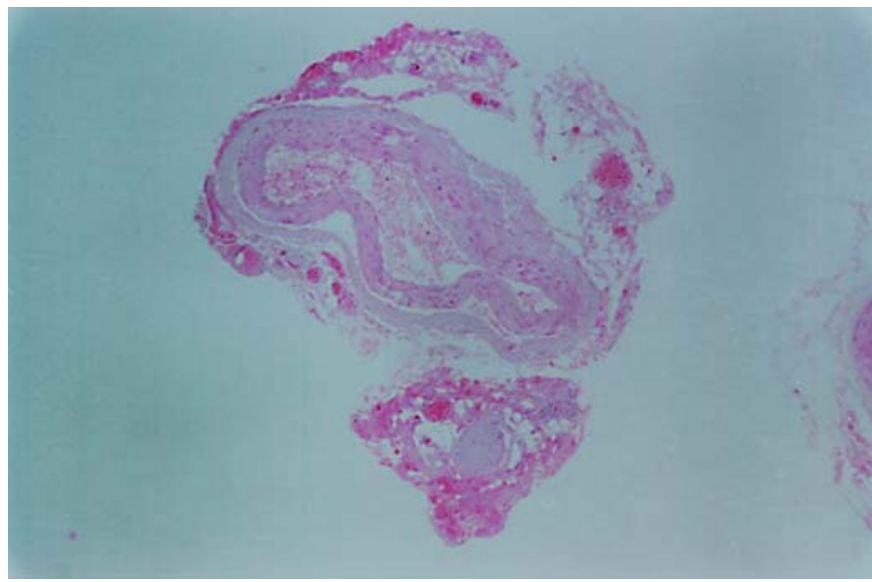

Fig. 3. Normal bronchial artery. H\&E. $\times 40$.

Moreover, there was no statistical significance among the different depositions of calcium along the inner elastic membrane in terms of diameter $(\mathrm{p}=0.27)$ or thickness $(\mathrm{p}=0.23)$. There was also no significant difference between the arteriosclerosis and the diameter of the vessels $(p=0.20)$. Interestingly, we found a relationship between the diameter of the vessels and TNM staging $(\mathrm{p}=0.031)$, and with the level of occlusion of the bronchial tree $(\mathrm{p}=$ 0.042 ).

Last but not least, there was no relationship between the diameter of the vessels, the thickness of the vessel wall and the presence of atherosclerotic lesions in the aorta (as demonstrated with a CT scan) and smoking status, alcohol

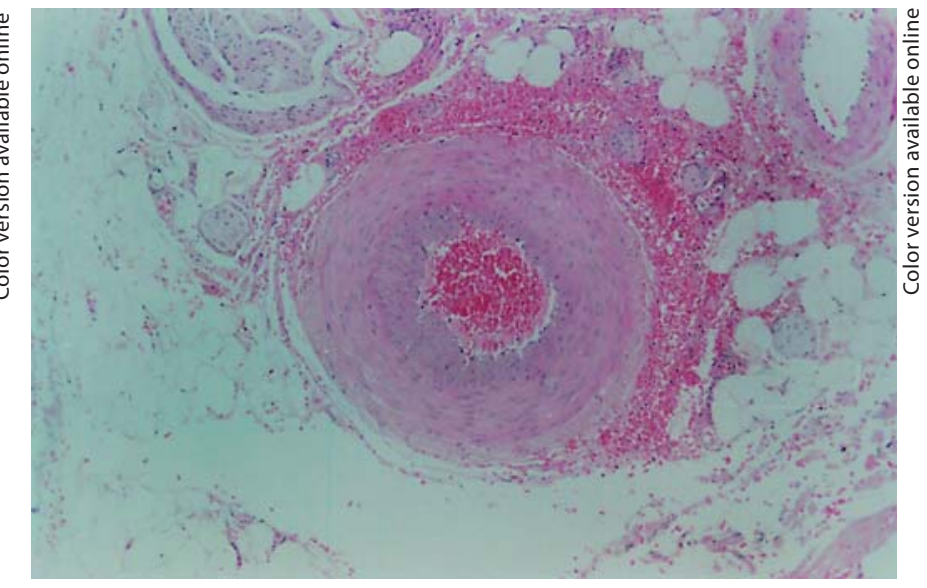

Fig. 2. Bronchial artery specimen with hypertensive-related changes. H\&E. $\times 100$.

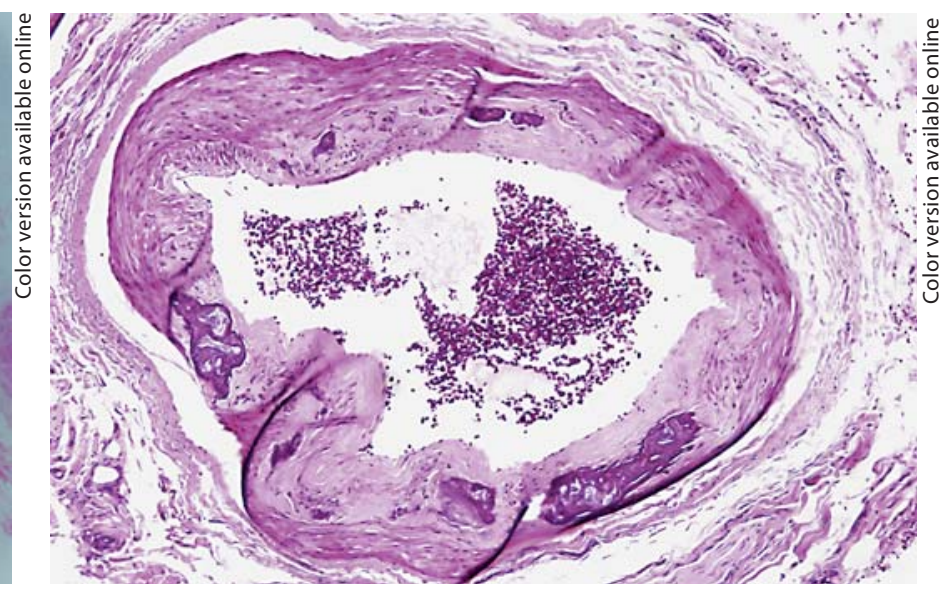

Fig. 4. Bronchial artery specimen with Mönckeberg's disease. H\&E. $\times 100$.

consumption, chronic obstructive airway disease, hypertension, diabetes mellitus, hyperlipidemia, CAD, peripheral vascular disease or family history of atherosclerosis.

\section{Comments}

The bronchial artery represents a muscular artery, while bronchial circulation is one of the dual blood supplies of the lungs. Although the importance of the bronchial arteries is evident in modern day thoracic surgery, there is no information about the prevalence of arteriosclerosis. The aim of our study was to investigate arterio- 
Table 3. Histopathological analysis of bronchial arteries: fibrosis

\begin{tabular}{|c|c|c|c|c|c|c|}
\hline & \multicolumn{3}{|c|}{ Fibrosis of the intima } & \multicolumn{3}{|c|}{ Fibrosis of the adventitia } \\
\hline & cases & diameter, $\mathrm{mm}$ & thickness, $\mathrm{mm}$ & cases & diameter, $\mathrm{mm}$ & thickness, $\mathrm{mm}$ \\
\hline Absent & 5 & $0.632 \pm 0.118$ & $0.102 \pm 0.018$ & 5 & $0.632 \pm 0.118$ & $0.102 \pm 0.018$ \\
\hline Mild & 9 & $0.938 \pm 0.315$ & $0.253 \pm 0.076$ & 17 & $0.828 \pm 0.289$ & $0.238 \pm 0.063$ \\
\hline Moderate & 20 & $0.846 \pm 0.339$ & $0.275 \pm 0.078$ & 18 & $0.955 \pm 0.327$ & $0.308 \pm 0.079$ \\
\hline Severe & 6 & $0.966 \pm 0.176$ & $0.296 \pm 0.097$ & - & - & - \\
\hline $\mathrm{p}$ value & & 0.166 & $<0.001$ & & 0.099 & $<0.001$ \\
\hline
\end{tabular}

Values are presented as the mean \pm SD. p values after ANOVA.

Table 4. Histopathological analysis of bronchial arteries: hypertrophy and calcium deposition

\begin{tabular}{|c|c|c|c|c|c|c|}
\hline & \multicolumn{3}{|c|}{ Hypertrophy of the media } & \multicolumn{3}{|c|}{ Calcium deposition (inner elastic membrane) } \\
\hline & cases & diameter, mm & thickness, mm & cases & diameter, $\mathrm{mm}$ & thickness, $\mathrm{mm}$ \\
\hline Absent & 5 & $0.632 \pm 0.118$ & $0.102 \pm 0.018$ & 10 & $0.837 \pm 0.394$ & $0.196 \pm 0.116$ \\
\hline Localized & - & & & 11 & $0.837 \pm 0.305$ & $0.271 \pm 0.084$ \\
\hline Mild & 11 & $0.788 \pm 0.270$ & $0.240 \pm 0.081$ & 16 & $0.802 \pm 0.170$ & $0.250 \pm 0.053$ \\
\hline Moderate & 19 & $0.892 \pm 0.273$ & $0.271 \pm 0.059$ & 3 & $1.325 \pm 0.248$ & $0.325 \pm 0.106$ \\
\hline Severe & 5 & $0.905 \pm 0.276$ & $0.346 \pm 0.099$ & - & - & \\
\hline $\mathrm{p}$ value & & 0.072 & 0.0001 & & 0.276 & 0.229 \\
\hline
\end{tabular}

Values are presented as the mean \pm SD. $p$ values after ANOVA.

sclerosis of the bronchial arteries, to associate it with arteriosclerotic risk factors and pulmonary disease and to validate its clinical significance, as there are no previous similar studies to our knowledge.

Samples were obtained during major lung or esophageal resection. Statistical analysis demonstrated no significant difference in the diameter of the bronchial arteries in respect to the site of resection $(p=0.37)$. The mean diameter of the vessels was $0.93 \mathrm{~mm}$, which is in agreement with the diameter of the bronchial arteries at the hilum [11]. Analysis has demonstrated that our sample was statistically adequate and representative since the expected relationship between the vessel wall thickness and the vessel diameter was found.

Histological analysis of the samples has demonstrated no evidence of invasion of the inner elastic membrane and eccentric reduction in the diameter of the vessel, namely typical atherosclerosis. Mönckeberg's disease, i.e. the presence of calcific medial sclerosis without invasion of the intima and rupture of the inner elastic membrane [12], was noted in only 1 patient (2.5\%). In addition, de- position of calcium in the inner elastic membrane was found in some of the arteries, which is more consistent with age-related changes [13]. In 2 cases, localized deposition of calcium was identified in the media. This is mainly related to the presence of arterial hypertension, in contrast to Mönckeberg's disease, where calcium deposition is noted in the inner elastic membrane [12]. Mönckeberg's disease typically involves small and medium size arteries in the breast, the splanchnic and peripheral circulation [14], as well as in the aorta and the soft tissue of the pharynx and larynx, but not of the coronaries $[15,16]$.

Because of the absence of previous similar studies, we compared our findings with other arterial systems, such as the intrathoracic internal mammary artery, the radial artery as the main representative of the limb arteries, the right gastroepiploic artery as the main representative of the splanchnic circulation, and the inferior epigastric artery. Their incidence of arteriosclerosis is based on many studies as they represent the usual conduits for coronary artery bypass grafting. It is worth noting that the internal mammary artery is a typical elastic artery, while the rest 
are muscular. Muscular arteries, like the right gastroepiploic artery and the radial artery, display a lesser amount of atherosclerosis compared with the inferior epigastric artery, due to the fact that the arteries supplying the upper part of the body and limbs display a lesser degree of atherosclerosis compared with the ones of the lower part of body and its limbs [17]. The right gastroepiploic artery exhibits the least degree of atherosclerosis of arteries of the splanchnic circulation $[18,19]$. In addition, it appears that the internal mammary artery is least likely to develop atherosclerosis compared with the rest of the arteries, followed by the radial artery [20,21]. Despite the fact that typical atherosclerosis barely affects these arteries and especially the internal mammary artery, Kane-ToddHall et al. [21] have demonstrated that the incidence of fibrotic sclerosis of the media in the internal mammary artery is much higher $(10 \%)$, as well as in the radial artery $(36 \%)$. Furthermore, the incidence of calcific sclerosis of the media was $9 \%$ for the radial artery and $0 \%$ for the internal mammary artery.

The presence of arteriosclerosis, in particular sclerosis of the media, was examined with all the biochemical and clinical factors related to its development. There was a borderline statistically significant relationship with the presence of metabolic syndrome $(\mathrm{p}=0.075)$. Surprisingly, none of the other risk factors were related, despite the fact that the family history, diabetes mellitus and age $>50$ years are amongst the strongest risk factors for the development of arteriosclerosis. Although Mönckeberg's disease can occur solely or in the presence of CAD, we found no such significant correlation $(\mathrm{p}=0.84)$.

As for malignant pulmonary disease, there was a significant relationship between the diameter of the vessel and the advanced stage of the disease $(p=0.031)$ and the proximal level of occlusion of the bronchial tree $(\mathrm{p}=$ 0.042). These findings indicate that proximal occlusion and/or the presence of mediastinal lymphadenopathy contribute to the increase in diameter of the bronchial arteries. This has already been shown in previous studies, which demonstrated that the diameter of the bronchial arteries increases in lung disease, especially in the presence of hemoptysis [22, 23]. Furthermore, no relationship was found between the diameter of the bronchial arteries and the presence of COPD ( $\mathrm{p}=0.91)$.

Despite the fact that our study is prospective, there can be statistical errors (type II) due to the small examined sample. Yet based at least on these results, it becomes clear that the bronchial artery exhibits a very low degree of arteriosclerosis, compared with the thoracic and the splanchnic circulation. We hypothesize that the bronchi- al arteries with a low degree of arteriosclerosis support the mediastinal status quo, contributing to all mediastinal structures and the heart, through the bronchial-coronary artery anastomoses, as we investigated and demonstrated in our previous study $[4,6,7]$. On this basis, this network may enable not only collateral coronary circulation in cases of CAD and thoracic aortic aneurysms, but may also assist the bronchial circulation in respiratory diseases. Further studies with simultaneous angiography of both circulations, especially in cases of CAD, may demonstrate these speculations in vivo. On the other hand, it would be of great interest to further investigate the underlying physiological background of the bronchial endothelium and circulation in order to identify the potential beneficial biochemical and/or immunological mechanisms that might contribute preventively against arteriosclerosis and might also have further therapeutic implications and implementations.

In conclusion, our study demonstrates a low incidence of arteriosclerosis and mainly calcific medial sclerosis (2.5\%) without the presence of endoluminal pathology in the bronchial arteries. There were no statistically significant associations between these findings and the presence of CAD or pulmonary disease. The bronchial resistance to arteriosclerosis might support the mediastinal status quo, contributing to all its structures, and might be indirect evidence of a different physiological function of the bronchial endothelium, which needs to be further investigated.
References
Respiration 2010;79:333-339
-1 Osaki T, Hanagiri T, Nakanishi R, Yoshino I, Taga S, Yasumoto K: Bronchial arterial infusion is an effective therapeutic modality for centrally located early-stage lung cancer: results of a pilot study. Chest 1999;115:14241428.

-2 Yoon W, Kim J, Kim Y, Chung T, Kang H: Bronchial and nonbronchial systemic artery embolization for life-threatening hemoptysis: a comprehensive review. Radiographics 2002;22:1395-1409.

- 3 Kamler M, Nowak K, Bock M, Herold U, Motsch J, Hagl S, Gebhard M, Jakob H: Bronchial artery revascularization restores peribronchial tissue oxygenation after lung transplantation. J Heart Lung Transplant 2004;23:763-766.

-4 Kotoulas C, Karnabatidis D, Kalogeropoulou C, Kokkinis K, Petsas T, Dougenis D: Anastomoses between bronchial and coronary circulation in a porcine model: computed tomographic and angiographic demonstration. Hellenic J Cardiol 2006;47: 206-210.

Kotoulas/Melachrinou/Konstantinou/ Alexopoulos/Dougenis 
5 Bjork L: Angiographic demonstration of extracardial anastomoses to the coronary arteries. Radiology 1966;87:274-277.

6 Iwasaki K, Kusachi S, Hina K, Murakami M, Matano S, Ohnishi N, Kondo N, Kita T, Sakakibara N: Coronary to bronchial artery anastomosis in patients with noncyanotic cardiopulmonary disease: report of seven cases. Can J Cardiol 1997;13:898-900.

7 Dupont P, Riquet M: The bronchial arteries. A review of their anatomy and their anastomoses with the coronary arteries. Surg Radiol Anat 1991;13:69-71.

$\checkmark 8$ Riquet M, Dupont P, Briere J, Weber S, Hidden G, Debesse B: Anastomoses between bronchial and coronary arteries: incidence of atheroma. Surg Radiol Anat 1991;13:349351.

9 Travis W, Colby T, Corrin B, Shimosato Y, Brambilla E, et al: Histological typing of lung cancer and pleural tumors; in WHO: International Histological Classifications of Tumors, ed 3. Geneva, Springer, 1999, pp 1-55.

-10 Mountain CF: Revisions in the International System for Staging Lung Cancer. Chest 1997; 111:1710-1717.

-11 Pump K: Distribution of bronchial arteries in the human lung. Chest 1972;62:447-451.
12 Mönckeberg J: Über die reine Mediaverkalkung der Extremitätenarterien und ihr Verhalten zur Arteriosklerose. Virchows Arch Pathol Anat 1902;171:141-167.

13 Nordborg C, Nordborg E, Petursdottir V, Fyhr I: Calcification of the internal elastic membrane in temporal arteries: its relation to age and gender. Clin Exp Rheumatol 2001; 19:565-568.

14 Kragel P, Aquino M, Fiorella R, Chapman J: Clinical, radiographic, and pathologic features of medical calcific sclerosis in the breast. South Med J 1997;90:518-521.

15 Couri C, da Silva G, Martinez J, Pereira Fde A, de Paula F: Mönckeberg's sclerosis - is the artery the only target of calcification? BMC Cardiovasc Disord 2005;5:34.

16 Doherty T, Fitzpatrick L, Inoue D, Qiao J, Fishbein M, Detrano R, Shah P, Rajavashisth T: Molecular, endocrine, and genetic mechanisms of arterial calcification. Endocr Rev 2004;25:629-672.

17 He G, Acuff T, Ryan W, Yang C, Mack M: Functional comparison between the human inferior epigastric artery and internal mammary artery. Similarities and differences. J Thorac Cardiovasc Surg 1995;109:13-20.
18 Van Son J, Smedts F, Yang C, Mravunac M, Falk V, Mohr F, He G: Morphometric study of the right gastroepiploic and inferior epigastric arteries. Ann Thorac Surg 1997;63: 709-715.

19 Suma H, Amano A, Horii T, Kigawa I, Fukuda S, Wanibuchi Y: Gastroepiploic artery graft in 400 patients. Eur J Cardiothorac Surg 1996;10:6-10.

20 Kaufer E, Factor S, Frame R, Brodman R: Pathology of the radial and internal thoracic arteries used as coronary artery bypass grafts. Ann Thorac Surg 1997;63:11181122.

21 Kane-ToddHall S, Taggart S, Clements-Jewery H, Roskell D: Pre-existing vascular disease in the radial artery and other coronary artery bypass conduits. Eur J Med Res 1999; 4:11-14.

22 Remy-Jardin M, Bouaziz N, Dumont P, Brillet P, Bruzzi J, Remy J: Bronchial and nonbronchial systemic arteries at multi-detector row CT angiography: comparison with conventional angiography. Radiology 2004;233: 741-749.

23 Moberg A: Anastomosis between extracardiac vessels to coronary arteries. Acta Radiol Diagn 1967;6:177-192. 